\title{
Right Adrenal Gland
}

National Cancer Institute

\section{Source}

National Cancer Institute. Right Adrenal Gland. NCI Thesaurus. Code C49325.

The adrenal gland located on the right side of the body. 